\title{
Raleio mecânico de frutos e flores em pessegueiros
}

\author{
Mechanical thinning of fruits and flowers in peach trees
}

\author{
Caroline Farias Barreto ${ }^{1 \star}$, Renan Navroski ${ }^{1}$, Jorge Atilio Benati ${ }^{1}$, José Francisco Martins Pereira ${ }^{2}$, \\ Marcelo Barbosa Malgarim 1 , Luis Eduardo Correa Antunes ${ }^{2}$
}

${ }^{1}$ Universidade Federal de Pelotas, Pelotas, RS, Brasil. Autor para correspondência: carol_fariasb@hotmail.com

${ }^{2}$ Embrapa Clima Temperado, Pelotas, RS, Brasil.

Submissão: 26/06/2019 / Aceite: 06/03/2020

\begin{abstract}
RESUMO
Técnicas que melhorem a eficiência do processo e reduzam o custo de operação são importantes ferramentas na redução dos custos. Dois dispositivos mecânicos foram utilizados para testar a eficiência no raleio de frutos e flores em pessegueiros e a influencia nas características produtivas e qualitativas dos frutos. O estudo foi realizado na cultivar Maciel em pomar comercial no município de Pelotas, Rio Grande do Sul, Brasil. Os tratamentos foram: raleio mecânico de flores com o equipamento Carpa Electro ${ }^{\circledR} \mathrm{e}$ "derriçadeira", raleio mecânico de frutos com o equipamento "derriçadeira" e raleio manual de frutos. 0 raleio mecânico associado ao repasse manual em pessegueiros pode ser uma alternativa viável ao raleio manual, pois reduz o tempo de execução desta prática. Os dois equipamentos podem ser utilizados para o raleio mecânico, pois não alteraram a produção por planta, o número de frutos por planta, a massa dos frutos, diâmetro dos frutos, as características físico-químicas e os compostos bioativos dos frutos.
\end{abstract}

PALAVRAS-CHAVE: compostos bioativos, custo de raleio, produtividade, Prunus persica, qualidade dos frutos, raleio manual.

\begin{abstract}
Techniques that improve process efficiency are important to reduce the operational cost of orchards. Two mechanical devices were tested to verify the efficiency of peach tree thinning and the influence on fruit production and quality characteristics. The experiment was conducted using the Maciel cultivar in a commercial orchard in Pelotas, Rio Grande do Sul, Brazil. The treatments consisted of mechanical flower blossom thinning using the Carpa Electro ${ }^{\mathrm{TM}}$ and "trimming" equipment; mechanical fruit thinning using the "trimming" equipment; and manual fruit thinning. The mechanical thinning associated with manual thinning of peach trees may be a viable alternative to manual thinning since it reduces the execution time. Both equipment can be used for mechanical thinning since they didn't change the production per plant, number of fruits per plant, fruit mass, fruit diameter, physicochemical characteristics, and bioactive compounds of the fruits.
\end{abstract}

KEYWORDS: bioactive compounds, thinning cost, productivity, fruit quality, Prunus persica, manual thinning.

As frutíferas de caroço apresentam florescimento abundante e elevada frutificação efetiva, produzindo uma quantidade excessiva de frutos e com baixo valor comercial (DESHMUKH et al. 2017, ASSIRELLI et al. 2018). Para reduzir essa carga de frutos, uma das práticas de manejo utilizada pelos produtores é o raleio de flores ou frutos, realizados de forma manual. Essa prática tem como objetivo melhorar a qualidade dos frutos e evitar alternância de produção (TURK et al. 2014, ASSIRELLI et al. 2018).

Nos pessegueiros, o raleio é realizado de forma manual entre 40 e 50 dias após a plena floração (OLIVEIRA et al. 2017). No entanto, essa é uma das práticas culturais mais onerosas na produção, pois é realizado em curto período de tempo e exige mão de obra qualificada para executá-la (BUSSI \& GENARD 2014, ASSIRELLI et al. 2018). Segundo a estimativa de março de 2017 da Companhia Nacional de Abastecimento (CONAB), a mão de obra representa $40,25 \%$ do custo total de produção de pêssegos no Brasil. O raleio manual de frutos pode levar em média 8 minutos por pessegueiro conduzido em sistema de vaso e com onze anos de idade (BARRETO et al. 2019), ocasionando em aumento de custos de produção de pêssegos (BUSSI \& GENARD 2014). 
$\mathrm{Na}$ tentativa de reduzir os custos de produção, outros métodos de raleio estão sendo estudados, incluindo produtos químicos e a remoção mecânica de flores e frutos (GIOVANAZ et al. 2016, BARRETO et al. 2018, ASSIRELLI et al. 2018). O raleio mecânico pode ser empregado para o cultivo de pessegueiros em virtude de ser uma prática rápida, através do uso de diferentes equipamentos e, assim, reduzir o custo e o tempo de trabalho (MARTIN-GORRIZ et al. 2011, SIMÕES et al. 2013, ASTEGGIANO et al. 2015).

$O$ raleio mecânico em pessegueiros pode ser realizado por meio de plataformas tratorizadas e dispositivos mecânicos manuais. Este tipo de raleio tem sido utilizado em diversos países como Espanha, Canadá, Itália e Estados Unidos (MARTIN et al. 2010, MILLER et al. 2011, MARTIN-GORRIZ et al. 2011, SAUERTEIG \& CLINE 2013, ASSIRELLI et al. 2018). Tendo em vista a falta de informações sobre o raleio mecânico em pessegueiros no Brasil, é necessário aprimorar os estudos para que a tecnologia possa ser aperfeiçoada e implementada de fato nos pomares brasileiros. Neste contexto, o presente trabalho teve como objetivo avaliar diferentes equipamentos para a realização do raleio mecânico de flores e frutos de pessegueiros.

O experimento foi conduzido na safra de 2017 em pomar comercial, no município de Pelotas, Rio

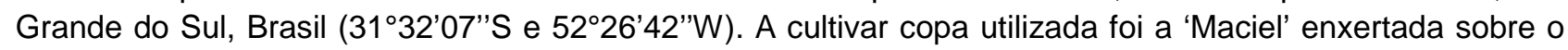
porta-enxerto 'Capdeboscq'. O pomar foi implantado no ano 2011, no espaçamento de 3,0m entre planta e $5,0 \mathrm{~m}$ na linha e com sistema de condução das plantas em vaso. A temperatura média, precipitação média e horas de frio durante o período de experimento foram coletadas da estação meteorológica da Embrapa Clima Temperadas na estação experimental da Cascata (Figura 1). No ano de 2017 foram registadas 198 horas de frio as quais foram calculadas com base nas temperaturas abaixo ou igual a $7,2{ }^{\circ} \mathrm{C}$.

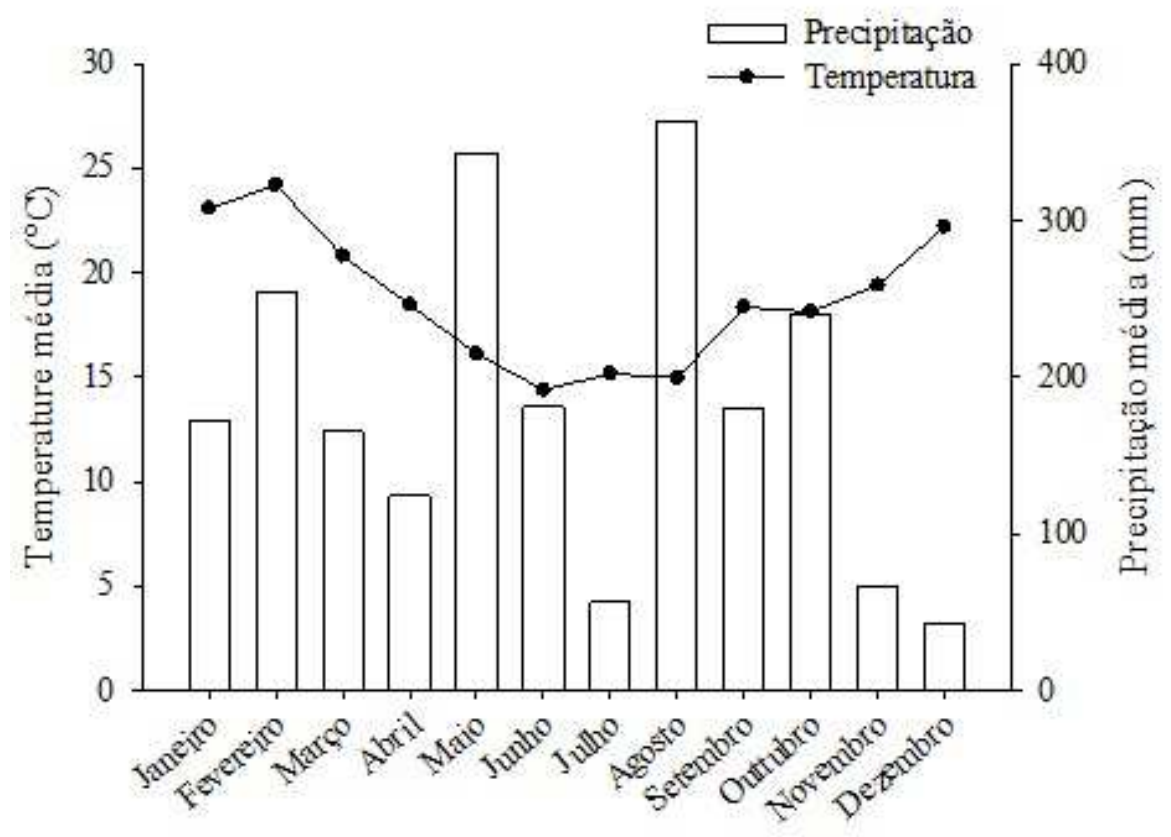

Figura 1. Temperatura e precipitação média mensal no ano de 2017 para o município de Pelotas, RS, Brasil. Figure 1. Average monthly precipitation and temperature in the year 2017 for the municipality of Pelotas, RS, Brazil.

O delineamento experimental foi em blocos ao acaso, com quatro tratamentos e quatro repetições de duas plantas por parcela. Os tratamentos foram: raleio manual dos frutos aos 40 dias após a plena floração (DAPF); raleio mecânico de flores na plena floração com o uso do equipamento Carpa Electro ${ }^{\circledR}$; raleio mecânico de flores na plena floração com o uso do equipamento "derriçadeira"; raleio mecânico dos frutos aos 40 DAPF com o uso do equipamento "derriçadeira". A plena floração foi considerada quando $50 \%$ das flores dos pessegueiros estavam abertas e o raleio manual dos frutos ocorreu quando estes estavam com aproximadamente $20 \mathrm{~mm}$ de diâmetro. Após a realização do raleio mecânico nos pessegueiros foi realizado o repasse do raleio de forma manual aos 40 DAPF.

O raleio mecânico foi efetuado pelo dispositivo manual Carpa Electro ${ }^{\circledR}$, que possui uma haste rotatória de $20 \mathrm{~cm}$ de comprimento, com anéis flexíveis de borracha que é ligado a uma bateria portátil (Figura 2A) e o equipamento "derriçadeira" da marca Sthil composta de um motor a gasolina, haste de 1,5 $\mathrm{m}$ e um par de garras de 0,39 $\mathrm{m}$ de comprimento (Figura 2B). O raleio manual foi realizado deixando de $10 \mathrm{a}$ 
$15 \mathrm{~cm}$ de distância entre os frutos. A plena floração dos pessegueiros ocorreu no dia 09 de agosto em 2017.
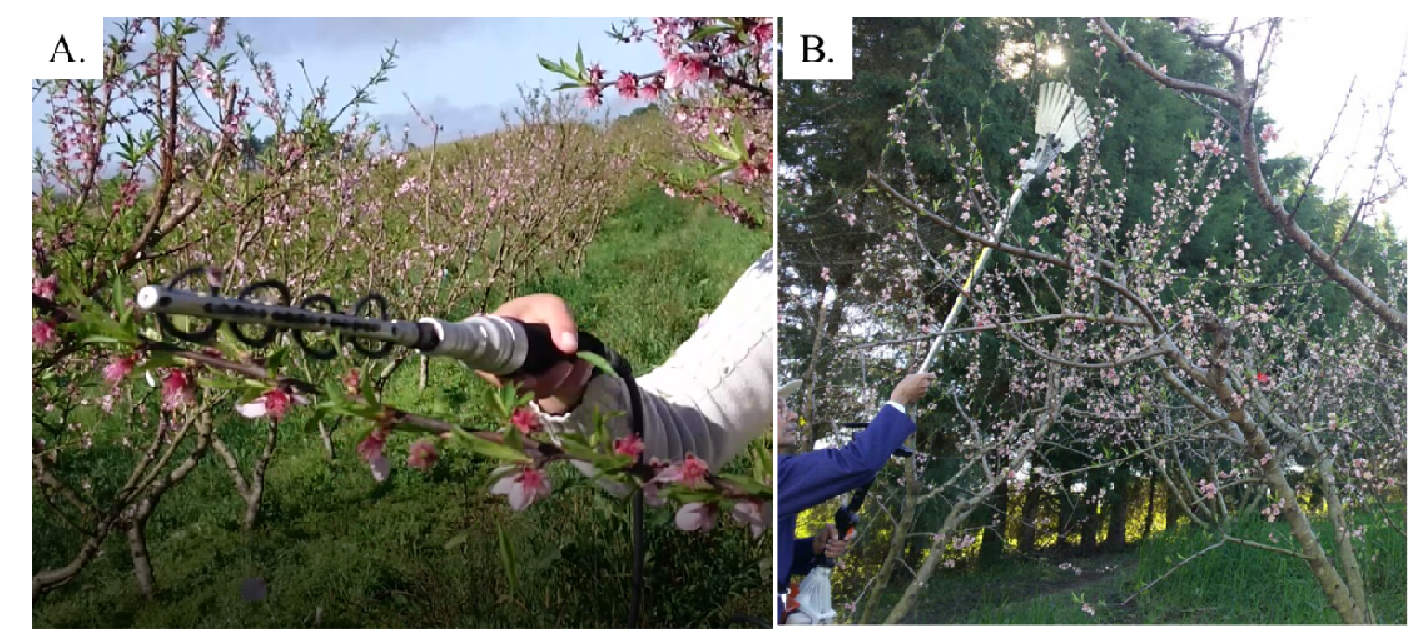

Figura 2. Equipamentos Carpa Electro ${ }^{\circledR}(A)$ e "derriçadeira" $(B)$ usados no raleio mecânico de flores e frutos de pessegueiros.

Figure 2. Carpa Electro ${ }^{T M}$ Equipment $(A)$ and "trimming" equipment $(B)$ used in the mechanical thinning of peach flowers and fruits.

Para avaliar a eficiência agronômica, analisou-se: porcentagem de queda de flores/frutos, determinada em seis ramos previamente selecionados aleatoriamente em cada planta pela contagem do número de flores/frutos antes e após a realização do raleio; tempo de raleio e repasse manual, determinado pelo tempo gasto na execução de cada tratamento, cronometrado com auxílio de relógio digital (min. planta $^{-1}$ ); tempo total de trabalho gasto, determinado pelo tempo de raleio + tempo de repasse manual (min. planta $^{-1}$ ); número de frutos por planta no momento da colheita (no frutos planta ${ }^{-1}$ ); produção por planta ( $\mathrm{Kg}$ planta $\left.^{-1}\right)$.

Após a colheita, foram selecionados aleatoriamente 50 frutos por repetição para avaliar a massa média dos mesmos (gramas); coloração da epiderme, realizada com auxílio do colorímetro marca Minolta CR- $300^{\circledR}$, com fonte de luz D65, onde se realizaram leituras de "L" (luminosidade), "a*", "b*" e a matiz ou tonalidade cromática representada pelo "ângulo hue"; à firmeza da polpa, medida com penetrômetro manual, marca TR TURONI-Italy, modelo $53205 \mathrm{com}$ ponteira de $8 \mathrm{~mm}$, em dois pontos opostos na região equatorial dos frutos sem casca, sendo os resultados expressos em Newtons; sólidos solúveis (SS), obtidos com o refratômetro digital da marca Atago ${ }^{\circledR}$, sendo os resultados expressos em ${ }^{\circ}$ Brix (AOAC 2005); acidez titulável (AT), quantificada em $10 \mathrm{~mL}$ de suco diluídos em $90 \mathrm{~mL}$ de água destilada e titulados Quimus pHmeter, sendo expressa em porcentagem de ácido cítrico (INSTITUTO ADOLFO LUTZ 2008).

Para determinação dos fitoquímicos da polpa dos pêssegos foram realizadas as seguintes análises: compostos fenólicos totais, determinados pelo método baseado na reação com o reagente Folin-Ciocalteau conforme o método adaptado de SINGLETON \& ROSSI (1965), sendo o resultado expresso em mg equivalente de ácido gálico em $100 \mathrm{~g}$ de amostra; atividade antioxidante, determinada pelo método do radical DPPH adaptado de BRAND-WILLIAMS et al. (1995) e os resultados expressos mg equivalente trolox $100 \mathrm{~g}^{-1}$ peso fresco.

Os dados foram submetidos à análise de variância através do teste $F(p \leq 0,05)$. Constatando-se significância estatística, os efeitos dos raleios foram comparados pelo teste de Tukey. A análise estatística foi realizada com o auxílio do programa Sisvar software, versão 5.6 (FERREIRA 2014).

O raleio mecânico com os equipamentos Carpa Electro ${ }^{\circledR}$ e "derriçadeira" proporcionaram a queda das flores de forma similar (Tabela 1). Esse percentual de queda de flores encontra-se na faixa reportada por SAUERTEIG \& CLINE (2013) e ASSIRELLI et al. (2018) que eliminaram de $42 \%$ a $75 \%$ das flores de pessegueiro no raleio mecânico. O raleio nos frutos com o equipamento "derriçadeira" obteve maior intensidade de raleio em comparação ao raleio manual de frutos (Tabela 1).

O raleio manual dos frutos necessitou do maior tempo para a execução (27,35 minutos), entretanto verificaram-se menores tempos no raleio mecânico na flor e no fruto com a "derriçadeira" (3,90 e 4,07 minutos) (Tabela 1). BUSSI \& GENARD (2014) verificaram tempo semelhante para o releio manual de pêssegos, levando entre 15 e 30 minutos por planta. MARTIN et al. (2010) também verificaram que o raleio manual exige maior tempo para execução em relação ao raleio mecânico, evidenciando que o raleio manual dos frutos encarece os custos de produção. 
Tabela 1. Porcentagem de queda de flores e frutos, tempo de raleio, tempo de repasse manual, tempo total gasto com raleio de pessegueiros 'Maciel' raleados manualmente e mecanicamente.

Table 1. Percentage of flower and fruit drop, thinning time, manual re-thinning time, total time spent with the manual and mechanical thinning of 'Maciel' peach trees.

\begin{tabular}{lccccc}
\hline Métodos de raleio & $\begin{array}{c}\text { Porcentagem } \\
\text { de queda de } \\
\text { flores } \\
(\%)\end{array}$ & $\begin{array}{c}\text { Porcentagem } \\
\text { de queda de } \\
\text { frutos } \\
(\%)\end{array}$ & $\begin{array}{c}\text { Tempo de } \\
\text { raleio }\end{array}$ & $\begin{array}{c}\text { Tempo de } \\
\text { repasse } \\
\text { manual } \\
\text { (min. planta }^{-1} \text { ) }\end{array}$ & $\begin{array}{c}\text { Tempo total } \\
\text { gasto com } \\
\text { raleio }\end{array}$ \\
\hline Raleio manual & - & $36,72 \mathrm{~b}$ & $27,35 \mathrm{a}$ & $0,00 \mathrm{~b}$ & $27,35 \mathrm{a}$ \\
RF Carpa Electro & $55,48^{\mathrm{ns}}$ & - & $10,92 \mathrm{~b}$ & $4,50 \mathrm{a}$ & $15,46 \mathrm{a}$ \\
RF "Derriçadeira" & 54,16 & - & $3,90 \mathrm{c}$ & $4,59 \mathrm{a}$ & $8,49 \mathrm{~b}$ \\
RFR "Derriçadeira" & - & $59,38 \mathrm{a}$ & $4,07 \mathrm{c}$ & $3,44 \mathrm{a}$ & $7,51 \mathrm{c}$ \\
\hline CV (\%) & 11,56 & 16,52 & 9,15 & 22,89 & 8,48 \\
p & 0,777 & 0,006 & 0,001 & 0,001 & 0,001 \\
\hline
\end{tabular}

Médias seguidas por letras minúsculas distintas, na mesma coluna, diferem entre si, pelo teste de Tukey, ao nível de $5 \%$ de probabilidade de erro. $n s$ = não significativo. $\mathrm{RF}=$ raleio de flores; $\mathrm{RFR}=$ raleio de frutos.

No repasse manual do raleio não houve diferenças no tempo de execução nas plantas raleadas mecanicamente (Tabela 1). O raleio mecânico na floração dos pessegueiros com o equipamento Carpa Electro $^{\circledast}$ reduziu em $43,47 \%$ o tempo total gasto com raleio quando comparado ao manual. Contudo, o tempo total gasto com o equipamento "derriçadeira" no raleio de flores e frutos reduziram em $68,95 \%$ e $72,54 \%$, respectivamente, em relação ao raleio manual dos frutos.

Verificou-se que, independente do equipamento, o raleio mecânico nos pessegueiros proporciona economia de tempo para a execução dessa prática. Esses resultados estão de acordo com MARTINGORRIZ et al. (2011), SAUERTEIG \& CLINE (2013) e ASSIRELLI et al. (2018) que evidenciam que o raleio mecânico de flores e frutos em pessegueiro reduz o tempo total de trabalho, em pomares na Espanha, Canadá e Itália.

No raleio mecânico de frutos com o equipamento "derriçadeira" foi possível realizar em média três plantas, enquanto se realiza uma planta no raleio manual. Portanto, a redução do tempo da execução do raleio é positiva, visto que há relação direta com a redução dos custos. Além do menor tempo de operação, os equipamentos testados nesse estudo podem ser utilizados em qualquer sistema de condução de plantas por serem dispositivos mecânicos manuais. Os equipamentos testados nesse estudo podem ser utilizados nas condições dos pomares brasileiros, mesmo que os pomares não tenham sido implantados visando a mecanizar o sistema de cultivo.

Em relação aos parâmetros produtivos do pessegueiro, a produção por planta, número de frutos por planta, a massa média dos frutos e diâmetro dos frutos não se observou diferenças entre o raleio mecânico e manual dos frutos (Tabela 2). Em outros estudos, o raleio mecânico dos pessegueiros não alterou a produção por planta (MARTIN-GORRIZ et al. 2011) e número de frutos por planta (MARTIN et al. 2010). Portanto, verifica-se a eficiência dos equipamentos testados neste estudo em reduzir o número de flores e frutos, mas sem alterar os índices produtivos.

Tabela 2. Produção por planta, número de frutos, massa dos frutos, diâmetro dos frutos de pessegueiros 'Maciel' raleados manualmente e mecanicamente.

Table 2. Production per plant, number of fruits, fruit mass, fruit diameter of 'Maciel' peach trees manually and mechanically thinned.

\begin{tabular}{lcccc}
\hline Métodos de raleio & $\begin{array}{c}\text { Produção por } \\
\text { planta }(\mathrm{Kg})\end{array}$ & $\begin{array}{c}\text { Número de } \\
\text { frutos }\end{array}$ & $\begin{array}{c}\text { Massa dos } \\
\text { frutos }(\mathrm{g})\end{array}$ & $\begin{array}{c}\text { Diâmetro dos } \\
\text { frutos (mm) }\end{array}$ \\
\hline Raleio manual & $110,98^{\mathrm{ns}}$ & $847^{\mathrm{ns}}$ & $129,43^{\mathrm{ns}}$ & $65,05^{\mathrm{ns}}$ \\
RF Carpa Electro & 99,23 & 788 & 128,76 & 64,85 \\
RF "Derriçadeira" & 96,26 & 632 & 155,02 & 66,75 \\
RFR "Derriçadeira" & 106,83 & 734 & 145,89 & 66,60 \\
\hline CV (\%) & 19,09 & 17,46 & 13,83 & 3,61 \\
p & 0,707 & 0,178 & 0,204 & 0,564 \\
\hline
\end{tabular}

Médias seguidas por letras minúsculas distintas, na mesma coluna, diferem entre si, pelo teste de Tukey, ao nível de $5 \%$ de probabilidade de erro. $n s$ = não significativo. RF = raleio de flores; RFR= raleio de frutos. 
Os parâmetros de qualidade dos pêssegos (físico-químicas e compostos bioativo) não modificaram quando provenientes de plantas raleadas mecanicamente e manualmente (Tabela 3). Segundo SAUERTEIG \& CLINE (2013) a firmeza e os sólidos solúveis dos frutos também não foram alterados quando se realizou o raleio mecânico em flores de pessegueiros. $O$ método de raleio não alterou a qualidade dos pêssegos neste estudo, deste modo, sugere-se que a qualidade pode variar devido a outros fatores, como cultivar (FERREIRA et al. 2016), porta-enxerto (BARRETO et al. 2017) irrigação, exposição à luz solar (ALCOBENDAS et al. 2013) e adubação (FERREIRA et al. 2016).

Tabela 3. Coloração da epiderme (CE), sólidos solúveis (SS), firmeza de polpa (FP), acidez titulável (AT), fenóis totais (FE) e atividade antioxidante (AA) de pessegueiros 'Maciel' raleados manualmente e mecanicamente.

Table 3. Coloration of the epidermis (CE), soluble solids (SS), pulp firmness (FP), titratable acidity (AT), total phenols (FE), and antioxidant activity (AA) of manually and mechanically thinned 'Maciel' peach trees.

\begin{tabular}{llllllc}
\hline \multicolumn{1}{c}{ Métodos de raleio } & $\begin{array}{c}\mathrm{CE} \\
\left({ }^{\circ} \mathrm{Hue}\right)\end{array}$ & $\begin{array}{c}\mathrm{SS} \\
\left({ }^{\circ} \mathrm{Brix}\right)\end{array}$ & $\begin{array}{c}\mathrm{FP} \\
(\mathrm{N})\end{array}$ & $\mathrm{AT}^{1}$ & $\mathrm{FE}^{2}$ & $\mathrm{AA}^{3}$ \\
\hline Raleio manual & $88,90^{\text {ns }}$ & $11,55^{\text {ns }}$ & $22,05^{\text {ns }}$ & $0,66^{\text {ns }}$ & $56,23^{\text {ns }}$ & $214,37^{\text {ns }}$ \\
RF Carpa Electro & 88,72 & 11,40 & 18,04 & 0,59 & 48,81 & 152,90 \\
RF "Derriçadeira" & 88,79 & 10,50 & 21,05 & 0,60 & 44,24 & 183,80 \\
RFR "Derriçadeira" & 87,31 & 11,65 & 20,13 & 0,47 & 58,50 & 214,28 \\
\hline CV (\%) & 1,27 & 6,92 & 13,42 & 14,71 & 20,11 & 17,64 \\
p & 0,205 & 0,196 & 0,247 & 0,367 & 0,242 & 0,071 \\
\hline
\end{tabular}

Médias seguidas por letras minúsculas distintas, na mesma coluna, diferem entre si, pelo teste de Tukey, ao nível de $5 \%$ de probabilidade de erro. $n s=$ não significativo. $\mathrm{RF}=$ raleio de flores; RFR= raleio de frutos ${ }^{1}$ porcentagem de ácido cítrico/100 g fruta; ${ }^{2} \mathrm{mg}$ equivalente ácido gálico $100 \mathrm{~g}^{-1}$ peso fresco. ${ }^{3} \mathrm{mg}$ equivalente trolox $100 \mathrm{~g}^{-1}$ peso fresco.

O raleio mecânico associado com o raleio manual nos pessegueiros demonstrou ser uma alternativa viável ao raleio manual de frutos e reduz o tempo de execução desta prática. Ambos os equipamentos testados nesse estudo podem ser utilizados para a realização do raleio em pessegueiros, pois não alteram os índices produtivos e qualidade dos frutos.

\section{AGRADECIMENTOS}

Ao Conselho Nacional de Desenvolvimento Científico e Tecnológico (CNPq) pela concessão das bolsas de estudo e a EMBRAPA pelo apoio em infraestrutura e logística.

\section{REFERÊNCIAS}

AOAC. 2005. Association of Official Analytical Chemistry. Official methods of analysis of the Association of Official Analytical Chemistry. 18.ed. Washington: AOAC.

ALCOBENDAS $R$ et al. 2013. Effects of irrigation and fruit position on size, colour, firmness and sugar contents of fruits in a mid-late maturing peach cultivar. Scientia Horticulturae 164: 340-347.

ASSIRELLI A et al. 2018. Evaluation of the detachment force needed for mechanical thinning of green peach fruits. Sustainability 10: 2291.

ASTEGGIANO $L$ et al. 2015. Bloom mechanical thinning improves fruit quality and reduces production costs in peach. Acta Horticulturae 1084: 389-394.

BARRETO CF et al. 2019. Raleio mecânico como alternativa no cultivo de pessegueiros Revista de Ciências Agrárias 42: 502-508.

BARRETO CF et al. 2018. Effect of chemical thinning using 6-benzyladenine (BA) on Maciel peach (Prunus persica L.). Australian Journal of Crop Science 12: 980-984.

BARRETO CF et al. 2017. Agronomic performance of the Maciel peach with different rootstocks. Semina: Ciências Agrárias 38: 1217-1228.

BRAND-WILLIAMS W et al. 1995. Use of a free radical method to evaluate antioxidant activity. Food Science and Technology 28: 25-30.

BUSSI C \& GENARD M. 2014. Thinning and pruning to overcome alternate bearing in peach trees. European Journal of Horticultural Science 79: 313-317.

CONAB. 2017. Companhia Nacional de Abastecimento. Custo de produção de pessegueiro. Disponível em: https://www.conab.gov.br/info-agro/custos-de-producao/planilhas-de-custo-de-producao/itemlist/category/405-

planilhas-de-custos-de-producao-culturas-permanentes?start=10. Acesso em: 29 abr. 2019.

DESHMUKH NA et al. 2017. Effect of thinning time and fruit spacing on fruit maturity, yield, size, peel colour and quality 
attributes of peach cv. Flordasun. Indian Journal of Horticulture 74: 45-50.

FERREIRA LV et al 2016. Qualidade de pêssegos submetidos à adubação nitrogenada. Revista Iberoamericana de Tecnología Postcosecha 17: 231-240.

FERREIRA DF 2014. Sisvar: a guide for its Bootstrap procedures in multiple comparisons. Ciência e Agrotecnologia 38: $109-112$

GIOVANAZ MA et al. 2016. Gibberellic acid reduces flowering and time of manual thinning in 'Maciel' peach trees. Revista Brasileira de Fruticultura 38: 1-10.

INSTITUTO ADOLFO LUTZ. 2008. Métodos químicos e físicos para análise de alimentos. 4.ed. São Paulo: Instituto Adolfo Lutz. 1020p.

MARTIN B et al. 2010. Post-bloom thinning of peaches for canning with hand-held mechanical devices. Scientia Horticulturae 125: 658-665.

MARTIN-GORRIZ B et al. 2011. Feasibility of peach bloom thinning with hand-held mechanical devices. Scientia Horticulturae 129: 91-97.

MILLER SS et al. 2011. Performance of mechanical thinners for bloom or green fruit thinning in peaches. Hortscience 46: 43-51.

OLIVEIRA PD et al. 2017. Heading of shoots and hand thinning of flowers and fruits on 'BRS Kampai' peach trees. Pesquisa Agropecuária Brasileira 52: 1006-1016.

SAUERTEIG KA \& CLINE JA. 2013. Mechanical blossom thinning of 'Allstar' peaches influences yield and quality. Scientia Horticulturae 160: 243-250.

SIMÕES MP et al. 2013. Monda mecânica de flores com equipamento electro'flor em pessegueiros da cultivar 'Rich Lady'. Revista de Ciências Agrárias 363: 297-302.

SINGLETON VL \& ROSSI JR JA. 1965. Colorimetry of total phenolic with phosphomolybdic-phosphotungstic acid reagents. American Journal of Enology and Viticulture 16: 144-158.

TURK BA et al. 2014. Tergitol as a possible thinning agent for peach cv. Redhaven. Horticultural Science 41: 49-54. 